\title{
Age-related changes in within- and between-channel gap detection using sinusoidal stimuli
}

\section{Document Version}

Final published version

Link to publication record in Manchester Research Explorer

\section{Citation for published version (APA):}

Heinrich, A., \& Schneider, B. A. (2006). Age-related changes in within- and between-channel gap detection using sinusoidal stimuli. The Journal of the Acoustical Society of America, 119(4), 2316-2326.

\section{Published in:}

The Journal of the Acoustical Society of America

\section{Citing this paper}

Please note that where the full-text provided on Manchester Research Explorer is the Author Accepted Manuscript or Proof version this may differ from the final Published version. If citing, it is advised that you check and use the publisher's definitive version.

\section{General rights}

Copyright and moral rights for the publications made accessible in the Research Explorer are retained by the authors and/or other copyright owners and it is a condition of accessing publications that users recognise and abide by the legal requirements associated with these rights.

\section{Takedown policy}

If you believe that this document breaches copyright please refer to the University of Manchester's Takedown Procedures [http://man.ac.uk/04Y6Bo] or contact uml.scholarlycommunications@manchester.ac.uk providing relevant details, so we can investigate your claim.

\section{OPEN ACCESS}




\title{
Age-related changes in within- and between-channel gap detection using sinusoidal stimuli
}

\author{
Antje Heinrich ${ }^{\text {a) }}$ and Bruce Schneider \\ Center for Research on Biological Communications Systems, University of Toronto, \\ Mississauga, Ontario, L5L 1C6, Canada
}

(Received 20 July 2005; revised 10 January 2006; accepted 18 January 2006)

\begin{abstract}
Pure tone gap stimuli with identical (within-channel) or dissimilar (between-channel) marker frequencies of 1 and $2 \mathrm{kHz}$ were presented to young and old listeners in a two-interval forced choice gap detection task. To estimate the influence of extraneous duration cues on gap detection, thresholds in the between-channel conditions were obtained for two different sets of reference stimuli: reference stimuli that were matched to the overall duration of the gap stimulus, i.e., two markers plus the gap, and reference stimuli that were fixed at the combined duration of the two markers excluding the gap. Results from within-channel conditions were consistent with previous studies, i.e., there were small but highly reliable age differences, smaller gap thresholds at longer marker durations, and an interaction between the two variables. In between-channel conditions, however, age differences were not as clear cut. Rather, the effect of age varied as a function of duration cue and was more pronounced when stimuli were matched for overall duration than when the duration of the reference tone was fixed. (C) 2006 Acoustical Society of America.
\end{abstract}

[DOI: 10.1121/1.2173524]

PACS number(s): 43.66.Mk, 43.66.Ba [JHG]

Pages: $2316-2326$

\section{INTRODUCTION}

\section{A. Within- and between-channel gap detection}

In a two-interval forced-choice gap-detection task the participant is asked to listen to two successively presented sounds and identify the one that contains a small period of silence, the gap. The sound containing the gap consists of three parts: the leading marker (the portion of the sound before the gap), the gap, and the lagging marker (the portion of the sound after the gap). The no-gap reference stimulus consists of the leading and lagging markers with no gap between them. When the two markers consist of pure tones, the frequencies of the two markers can either be the same or different. Numerous studies have shown that gap detection thresholds are sensitive to the spectral similarities of the markers bounding the gap. In general, gap detection thresholds tend to increase and then asymptote, as the spectral difference between the leading and lagging markers becomes greater (Grose et al., 2001; Formby et al., 1998a; Formby and Forrest, 1991; Divenyi and Danner, 1977).

Several mechanisms have been suggested to account for this result (e.g., Formby and Forrest, 1991; Formby et al., 1996; Phillips et al., 1998, 1997; Fitzgibbons et al., 1974). All of these accounts assume that, in the first stage of auditory processing, the signal is processed by a bank of auditory filters, with each filter defining a separate auditory channel. Hence, when the two markers are presented to the same ear and are identical or nearly identical with respect to frequency content, then both markers are processed by the same auditory channel, and the detection of a gap can be based on

\footnotetext{
${ }^{a)}$ Present address: MRC Cognition and Brain Sciences Unit, 15 Chaucer Road, Cambridge, UK. Electronic mail: antje.heinrich@mrc-cbu.cam.ac.uk
}

within-channel comparisons. On the other hand, when the two markers differ substantially with respect to their frequency content and/or are presented to opposite ears, the detection of a gap has to involve the integration and/or comparison of events across two or more auditory channels (between-channel comparisons).

\section{B. Age-related changes in gap detection}

Comparatively few studies have investigated the influence of age on gap detection. The results to date suggest that the effect of age on gap detection thresholds differs for within- and between-channel tasks. In within-channel tasks age differences in threshold are generally small and related to marker duration. Schneider and Hamstra (1999), for instance, tested young and old listeners in a gap detection task where both markers were $2-\mathrm{kHz}$ pure tones. The duration of the markers varied in different conditions. They found that whereas there were considerable age differences when marker durations were short, these differences completely vanished for the longest durations. This result integrated previously conflicting evidence from Moore et al. (1992) and Schneider et al. (1994). Specifically, whereas Moore and colleagues did not find age differences in a within-channel gap detection task when marker durations were large, Schneider et al. (1994), using very short durations, found that thresholds for older listeners were generally larger and more variable than for young listeners.

A number of factors have been ruled out as having a direct influence on the age effect in within-channel gap detection. For instance, Schneider et al. (1998) demonstrated that age differences are probably not due to either differences in off-frequency processing or longer integration times of the temporal window in older listeners. Moreover, several stud- 
ies were unable to find any association between audiometric and gap thresholds, thus indicating that peripheral hearing loss has no direct influence on gap detection thresholds (e.g., Moore et al., 1989; Schneider et al., 1994; Schneider and Hamstra, 1999; Moore et al., 1992; but also see Lutman, 1991 , for a contrary position). One mechanism that has been suggested to contribute to the age effect is neural adaptation. Schneider and Hamstra (1999) argue that the fact that age differences are closely linked to marker duration suggests that there are differences in neural adaptation and recovery in response to markers of different lengths.

Lister and colleagues were among the first to examine the effects of age and hearing status in between-channel gap detection tasks (Lister et al., 2000, 2002; Roberts and Lister, 2004). In a first study they tested listeners with and without hearing loss in a gap duration discrimination task in which the two stimuli could be distinguished by the duration of the gap rather than its presence. The rationale for inserting a small (1 ms) gap in the reference stimulus was to ensure that both intervals contained similar gating transients. The stimuli were narrow-band noises. In a posthoc analysis Lister et al. (2000) tested the effect of age on gap threshold and found differences in threshold for young and old adults. Specifically, they found a steeper increase in threshold for older than for younger listeners as frequency disparity increased. Moreover, consistent with within-channel results, hearing status did not significantly influence gap thresholds. Rather, younger listeners, including those with a severe hearing impairment, always had smaller thresholds compared to even minimally impaired older adults. Hence, the study suggested that in between-channel tasks, like in within-channel tasks, reduced temporal processing in older adults is independent of peripheral hearing loss. In a second study, Lister et al. (2002) investigated the matter in greater detail and tested three groups of young, middle-aged, and older adults, respectively. The study showed again that thresholds increase more sharply with increasing frequency disparity for older listeners, a result that was confirmed by Roberts and Lister (2004). Hence, the effect of age is more apparent for frequencydisparate stimuli. Lister et al. (2002) speculate that the effect is caused by the fact that perceptual channels are more sharply tuned in older adults, presumably permitting them to use between-channel processing more effectively than younger adults for smaller frequency disparities.

Others have suggested that the increased age effect for between- compared to within-channel tasks can be linked to increased task complexity (Pichora-Fuller, 2003; PichoraFuller et al., 2006), an interpretation in concordance with results of a study by Snell (1997) that used more complex low-pass noise stimuli embedded into modulated and unmodulated noise maskers. The results of the Snell study showed a robust age effect for all conditions, even when the noise stimuli were presented in quiet and even though puretone thresholds at the test frequencies were comparable for young and old listeners. Moreover, adding a noise floor to the gap stimuli further increased age differences.

In summary, age differences in gap detection in withinchannel tasks depend on marker duration and may point to differences in neural adaptation. Age differences are ampli- fied in between-channel conditions and appear to be related to the difficulty in integrating or comparing information across channels. Finally, age differences appear to increase as the spectral and temporal complexity of the markers is increased. One objective of the study was to determine if age differences in between-channel gap detection can be minimized when stimulus complexity is reduced to a minimum. Hence, the present study determined gap detection thresholds for tonal markers ( 1 and $2 \mathrm{kHz}$ ) for both within- and between-channel conditions in young and old adults.

\section{Extraneous temporal and spectral cues to gap detection}

Extraneous cues, only indirectly related to the size of the gap, can affect between-channel gap detection. For instance, when the marker durations of the leading and lagging markers in both the gap and no-gap stimuli are held constant, the overall duration of the gap stimulus is longer than that of the no-gap reference stimulus by the amount equal to the size of the gap. Thus, the listener could be using overall duration to discriminate between gap and no-gap stimuli. Alternatively, when the overall durations of gap and no-gap stimuli are kept constant, the duration of each marker in the reference stimulus is longer than the duration of each marker in the gap stimulus. Hence, the listener could be performing the task based on the duration of the markers rather than on the perception of a gap. Differences in marker duration between gap and no-gap stimuli, however, are only effective for stimuli with dissimilar frequencies in leading and lagging marker because when the frequency content is identical in both markers, the listener cannot tell when the leading marker in the reference stimulus ends and the lagging marker begins. Hence, for within-channel tasks, equating overall duration removes any possibility that the task is based on marker duration.

A number of studies (Formby and Forrest, 1991; Formby and Muir, 1989; Penner, 1977) have demonstrated that listeners do indeed use extraneous duration cues to aid gap detection which in turn can lead to an underestimation of the true gap detection threshold. However, these studies also show that not all listeners use these duration cues to the same extent and that different listeners may use different cues or no extraneous cues at all. Support for the notion that duration cues, especially overall duration cues, could possibly modulate gap detection accuracy also comes from a different line of research. Bergeson et al. (2001) investigated duration discrimination for pure tones and found that for young adults the Weber fraction for a $20-\mathrm{ms}$ tone duration is 0.6, decreasing to 0.3 at $200 \mathrm{~ms}$. Thus, given an overall marker duration of $40 \mathrm{~ms}$ in the reference stimulus, duration differences could become noticeable when the target exceeds $64 \mathrm{~ms}$ in overall duration; this equals a gap size of $24 \mathrm{~ms}$ for younger adults. For older adults the Weber fraction is 1.3, and duration differences would only be noticeable at a much higher level (92 ms). Gap thresholds of over $20 \mathrm{~ms}$ are not unreasonable for young adults, and gap durations larger than $52 \mathrm{~ms}$ have been observed in older adults, especially for between-channel conditions. 
Whereas Formby and Forrest (1991) as well as Penner (1977) aimed to minimize duration cues in their studies by randomizing the duration of each marker independently, we chose to estimate their influence instead. Therefore, we included two sets of between-channel conditions that were specifically constructed to estimate the effects of overall and relative marker duration cues. In the first set of betweenchannel conditions, the overall duration of the reference stimulus matched that of the target. Hence, the individual durations of the two markers in the no-gap stimulus were longer than those in the gap stimulus. In a second set of conditions individual marker duration was kept constant between gap and no-gap stimuli, so that the overall duration of the no-gap stimulus was shorter than that of the gap stimulus.

Another possible cue to the presence of a gap is the spectral splatter that arises from the termination of the first marker and the onset of the second marker. If the amount of spectral splatter varies with gap duration, then spectral splatter could be used to determine the presence or absence of a gap. To minimize the effects of spectral splatter we constructed each marker by multiplying a pure tone by an envelope consisting of a sum of Gaussian envelopes, with the separation between the means of each successive Gaussian envelope equal to one standard deviation unit. This has been shown to minimize spectral cues in within-channel gap detection for short duration stimuli (Schneider and Hamstra, 1999, Schneider et al., 1994). In the Appendix, we show that the likelihood that the detection of a gap in between-channel conditions is based on spectral splatter is negligible. Hence, we can be reasonably certain that the gap detection thresholds observed here are based on temporal and not spectral cues.

In the present study we compared gap detection performance for young and old listeners in within- and betweenchannel tasks using two different marker durations (10 and $20 \mathrm{~ms}$ ). For within-channel tasks, Schneider and Hamstra (1999) found that the size of the age difference decreased as marker duration increased. For between-channel tasks, several studies suggested that age differences increase for more complex stimuli. Our first objective was to measure the extent of age differences for between-channel stimuli when stimulus complexity was reduced to a minimum. A second objective was to see if age differences also decreased as a function of marker duration for between-channel comparisons. Lastly, we sought to estimate the influence of extraneous duration cues on gap detection performance for young and old listeners in between-channel conditions.

\section{METHODS}

\section{A. Participants}

The final sample consisted of 24 young (mean age: 21.3 years; s.d.: $1.81 ; 14$ females) and 24 older listeners (mean age: 71.5 years; s.d.: $4.85 ; 12$ females). Three young listeners had to be replaced because they were not available for all experimental sessions, and three older listeners had to be replaced because they were not able to perceive the gap in between-channel conditions even with the longest duration. All listeners (with the exception of one in the older age
TABLE I. Mean and standard deviation (SD) of hearing thresholds in $\mathrm{dB}$ HL (ANSI, 1989) for nine pure-tone frequencies in the left (test) ear for young and old listeners. The superscript letters denote significant differences in hearing level between young and old listeners.

\begin{tabular}{lccccc}
\hline \hline & \multicolumn{2}{c}{ Young } & & \multicolumn{2}{c}{ Old } \\
\cline { 2 - 3 } \cline { 5 - 6 } Frequency in kHz & Mean & SD & & Mean & SD \\
\hline 0.25 & 9.79 & 4.54 & & $14.17^{\mathrm{a}}$ & 6.70 \\
0.5 & 4.38 & 3.40 & & $10.00^{\mathrm{b}}$ & 6.76 \\
1 & 3.13 & 6.05 & & $7.92^{\mathrm{a}}$ & 6.58 \\
1.5 & 3.33 & 6.54 & & $10.21^{\mathrm{b}}$ & 8.91 \\
2 & 3.33 & 5.45 & & $10.00^{\mathrm{b}}$ & 9.33 \\
3 & 0.83 & 6.86 & & $15.42^{\mathrm{b}}$ & 8.84 \\
4 & 3.13 & 5.86 & & $23.33^{\mathrm{b}}$ & 14.27 \\
6 & 11.25 & 9.12 & & $34.79^{\mathrm{b}}$ & 17.78 \\
8 & 6.04 & 7.07 & & $41.67^{\mathrm{b}}$ & 18.69 \\
\hline \hline
\end{tabular}

$\overline{\bar{a} p<0.05 \text {. }}$

${ }^{\mathrm{b}} p<0.01$.

group) had hearing thresholds at 1 and $2 \mathrm{kHz}$ in the test (left) ear that were $\leqslant 25 \mathrm{~dB}$ HL. One older listener had a hearing threshold of $35 \mathrm{~dB} \mathrm{HL}$ at $2 \mathrm{kHz}$. Thresholds at the two test frequencies were significantly higher for older than they were for younger adults, and the extent of this age difference increased with increasing frequency. Audiometric thresholds for a range of frequencies in the test (left) ear are displayed in Table I. The older adults could be characterized as having at most a slight hearing loss at the test frequencies (Clark, 1981) and as being in the early stages of presbyacusis.

\section{B. Stimuli}

The stimuli were digitally generated with a sampling rate of $20 \mathrm{kHz}$ and converted to analog form using a 16-bit Tucker Davis Technology (TDT) digital-to-analog converter. Various techniques were used in an effort to minimize the spectral splatter at the on- and off-set of the stimuli. Firstly, amplitude envelopes for gap and no-gap sounds were constructed by summing a series of Gaussian functions, spaced one standard deviation apart, with a standard deviation of $1 \mathrm{~ms}$ (Fig. 1). The sum of the envelopes formed a flat top with ogival rise and decay times. Secondly, all of the gap durations tested preserved the phase of the sinusoidal stimuli. Gaps were created by placing the mean of the first Gaussian in the second marker, an integer number of ms after the mean of the last Gaussian in the first marker. Hence, the smallest possible gap occurred when the first Gaussian in the second marker followed $2 \mathrm{~ms}$ after the last Gaussian in the first marker. However, pilot testing had revealed that this gap was perceivable by most of the young listeners in within-channel conditions. In order to create smaller energy dips for withinchannel conditions, we filled the smallest gap with a reduced-amplitude Gaussian instead of completely omitting it. Now, the gap was detected as a more or less shallow dip in the stimulus envelope. Originally, gap durations were measured as the distance between the $\frac{1}{2}$-power point at the end of the first marker to $\frac{1}{2}$-power point at the beginning of the second marker. In the case of filled-in Gaussians, however, the amplitude envelope did not decay to zero, but, instead, 


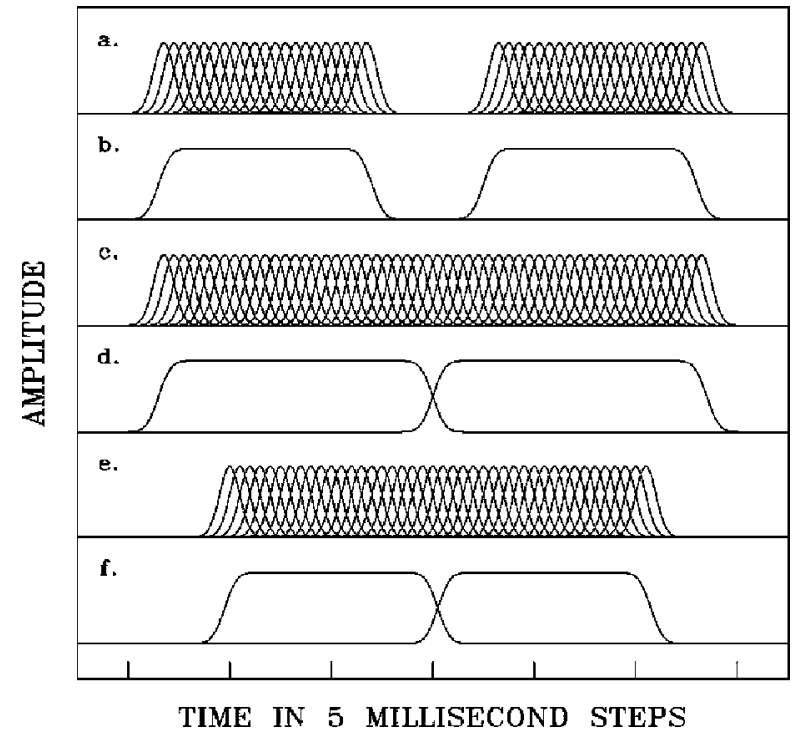

FIG. 1. (a) The Gaussian envelopes used to create two 20-ms markers (each marker consists of 21 Gaussians) with a 13-ms gap between the two markers. The Gaussian envelopes are spaced $1 \mathrm{~ms}$ apart, and there is a 13-ms gap between the last Gaussian in the leading marker and the first Gaussian in the lagging marker. (b) The two envelopes formed by summing the Gaussians in (a). (c) The Gaussians used to form the two marker envelopes of the no-gap stimulus that is equivalent in total duration to the 13-ms gap stimulus shown in (a) and (b). There are 27 Gaussians in the leading marker and 27 Gaussians in the lagging marker, with $1 \mathrm{~ms}$ separating the last Gaussian in the leading marker and the first Gaussian in the lagging marker. (d) The two envelopes formed by summing the Gaussians in (c). (e) The Gaussians used to form the envelopes of the no-gap stimulus whose marker durations are the same as in (a). There are 21 Gaussians in the first marker and 21 Gaussians in the lagging marker with $1 \mathrm{~ms}$ separating the last Gaussian in the leading marker and the first Gaussian in the lagging marker. (f) The two envelopes formed by summing the Gaussians in (e).

the sum of the two markers began to affect the $\frac{1}{2}$-power points, making these points unusable as points of reference. To avoid this problem when analyzing data, we used the area difference between the gap and the no-gap amplitude envelope functions to index gap duration. All analyses were conducted on the area difference between gap and no-gap envelope. However, in describing the data we converted the area measures back into equivalent gap durations. ${ }^{1}$

The leading and lagging marker envelopes were then multiplied by tones of 1 and $2 \mathrm{kHz}$. In the within-channel conditions, both leading and lagging marker were multiplied by the same $1-$ or $2-\mathrm{kHz}$ tone, respectively. In the betweenchannel conditions, leading and lagging markers were multiplied by different frequencies, either 1 or $2 \mathrm{kHz}$. The resulting product was normalized in such a way that all stimuli, gap and no-gap, contained the same amount of energy (Fig. 1). The marker duration for the gap stimuli was kept constant within each condition at 10 or $20 \mathrm{~ms}$, respectively. Two different sets of between-channel conditions were tested: a set where the overall duration of the no-gap stimulus matched that of the gap stimulus, and a set of conditions where the duration of the no-gap stimulus was fixed at the combined marker duration of leading and lagging markers, i.e., at either 20 or $40 \mathrm{~ms}$. Stimuli matched for overall duration were also used in all within-channel conditions. Note that under conditions of matched duration, the reference stimuli were constructed in such a way that they matched gap stimuli in over- all duration for odd gap durations (e.g., $9 \mathrm{~ms}$ ) and exceeded them in overall duration by $1 \mathrm{~ms}$ in cases of even gap sizes (e.g., $8 \mathrm{~ms}$ ). The sound pressure level of the pure tones before they were multiplied by the marker envelopes was $70 \mathrm{~dB}$ SPL. After the pure tones were multiplied by the amplitude envelopes, they were rescaled so that the total energy in a stimulus was equated across all conditions. Hence, there were no energy differences between gap and no-gap stimuli. Stimuli were presented to the left ear over TDH-49 earphones in a single-wall sound-attenuating booth.

\section{Psychoacoustic procedure}

A three-down, one-up tracking procedure was used in a 2AFC paradigm to determine the $79.4 \%$ accuracy point on the psychometric function (Levitt, 1971). At the beginning of each run, the gap size was set to its maximum value, $31 \mathrm{~ms}$ in within-channel conditions and $61 \mathrm{~ms}$ in between-channel conditions. In each trial, which was initiated by a button press, a gap and a no-gap stimulus were presented, randomly assigned to one of two 500-ms-long stimulus intervals, separated by $100 \mathrm{~ms}$. The task was to indicate which of the two intervals contained the gap stimulus. After three consecutive correct responses the gap size of the stimulus was decreased. After each incorrect response the gap size was increased. The size of the increment or decrement in gap was halved after each reversal until a minimum step size of $1 \mathrm{~ms}$ was reached. Each stimulus began $100 \mathrm{~ms}$ into the interval.

Participants indicated which interval they thought contained the gap by pressing one of two buttons. Lights on the button box indicated the beginning and the length of each interval and also provided immediate feedback to the subject about the accuracy of their response. Each run was ended after 12 reversals. The gap-detection threshold for the condition was defined as the average of the last eight reversals. Participants cycled through all conditions a total of four times. The gap threshold for each condition was defined as the geometric mean of the thresholds of the four runs. All listeners came in for three successive sessions of $1.5 \mathrm{~h}$ each; thresholds for within-channel conditions were obtained in session 1, for between-channel conditions with the reference stimulus matched in total duration in session 2, and for between-channel conditions with fixed marker durations in session 3. Testing within each session was counterbalanced for frequency content of the leading marker and marker duration between listeners. The testing was self-paced and initiated by a button press. Listeners were not given practice trials prior to data collection. Table II details all conditions tested in the study.

\section{RESULTS}

\section{A. Within-channel gap detection}

Figure 2 displays mean gap thresholds in milliseconds for young and old listeners in all four within-channel conditions. The thresholds are generally very small: between 0.9 and $1.6 \mathrm{~ms}$ for young listeners and 1.2 and $2.5 \mathrm{~ms}$ for older listeners. Moreover, the data show that the shorter marker duration $(10 \mathrm{~ms})$ generally led to longer gap thresholds. 
TABLE II. Stimulus conditions used in the study. Markers varied in frequency similarity and frequency order. Each condition was run a total of four times.

\begin{tabular}{|c|c|c|}
\hline Session no. & $\begin{array}{l}\text { Marker duration } \\
(\mathrm{ms})\end{array}$ & $\begin{array}{c}\text { Frequency of } \\
\text { leading and lagging } \\
\text { markers }\end{array}$ \\
\hline \multirow{4}{*}{ (1) Within-channel } & 10 & $1 \mathrm{kHz}, 1 \mathrm{kHz}$ \\
\hline & & $2 \mathrm{kHz}, 2 \mathrm{kHz}$ \\
\hline & 20 & $1 \mathrm{kHz}, 1 \mathrm{kHz}$ \\
\hline & & $2 \mathrm{kHz}, 2 \mathrm{kHz}$ \\
\hline \multirow{4}{*}{$\begin{array}{l}\text { (2) Between-channel, } \\
\text { matched reference } \\
\text { stimulus }\end{array}$} & 10 & $1 \mathrm{kHz}, 2 \mathrm{kHz}$ \\
\hline & 10 & $2 \mathrm{kHz}, 1 \mathrm{kHz}$ \\
\hline & 20 & $1 \mathrm{kHz}, 2 \mathrm{kHz}$ \\
\hline & 20 & $2 \mathrm{kHz}, 1 \mathrm{kHz}$ \\
\hline \multirow{4}{*}{$\begin{array}{l}\text { (3) Between-channel, } \\
\text { fixed reference } \\
\text { stimulus }\end{array}$} & \multirow{2}{*}{10} & $1 \mathrm{kHz}, 2 \mathrm{kHz}$ \\
\hline & & $2 \mathrm{kHz}, 1 \mathrm{kHz}$ \\
\hline & \multirow{2}{*}{20} & $1 \mathrm{kHz}, 2 \mathrm{kHz}$ \\
\hline & & $2 \mathrm{kHz}, 1 \mathrm{kHz}$ \\
\hline
\end{tabular}

Lastly, thresholds appear to be higher for $2-\mathrm{kHz}$ than for $1-\mathrm{kHz}$ markers and older listeners generally exhibited larger thresholds than young listeners.

A 2 marker duration $(10 \mathrm{~ms}, 20 \mathrm{~ms})$ by 2 marker frequency $(1 \mathrm{kHz}, 2 \mathrm{kHz})$ by 2 age group (young, old) mixed measures ANOVA with marker duration and marker frequency as within-subject variables and age as a betweensubjects variable supported the graphical results. ${ }^{2}$ The ANOVA revealed main effects of marker duration $[F(1,46)$ $=74.25, p<0.0001](20$-ms markers led to smaller thresholds than 10-ms markers), frequency $[F(1,46)=70.78, p$ $<0.0001]$ ( $2 \mathrm{kHz}$ stimuli resulted in larger overall thresholds), and age $[F(1,46)=12.28, p<0.001]$ (young listeners generally achieved smaller thresholds). Moreover, the interaction of marker duration and age reached significance $[F(1,46)=4.52, p<0.05]$. This interaction indicated that the age difference was more pronounced for the $10-\mathrm{ms}$ com-

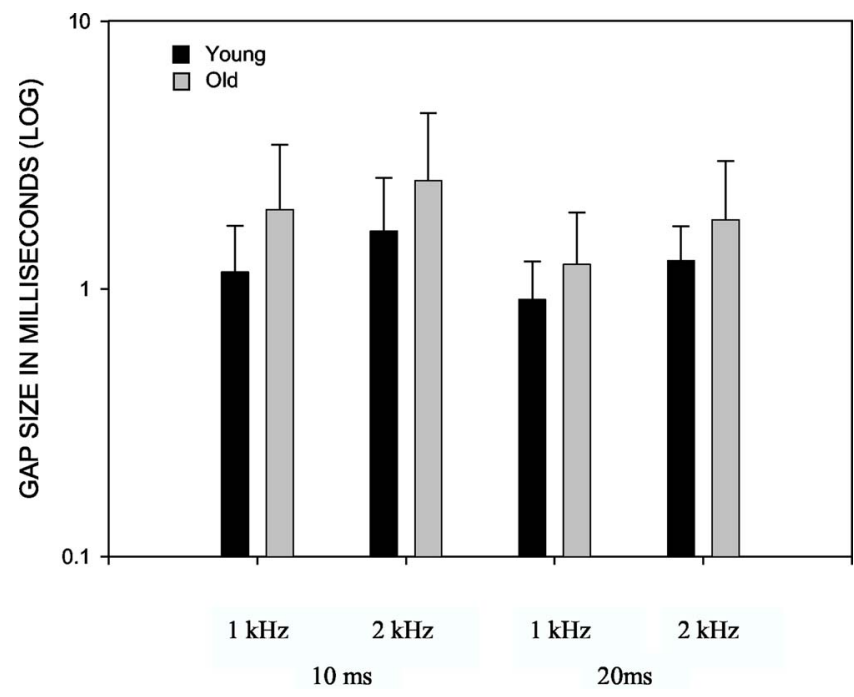

FIG. 2. Mean gap detection thresholds for young (black) and old (gray) listeners for 10- and 20-ms markers of identical frequency (within channel). The stimulus frequency of both markers was either 1 or $2 \mathrm{kHz}$. Error bars depict 1 standard deviation above the mean.
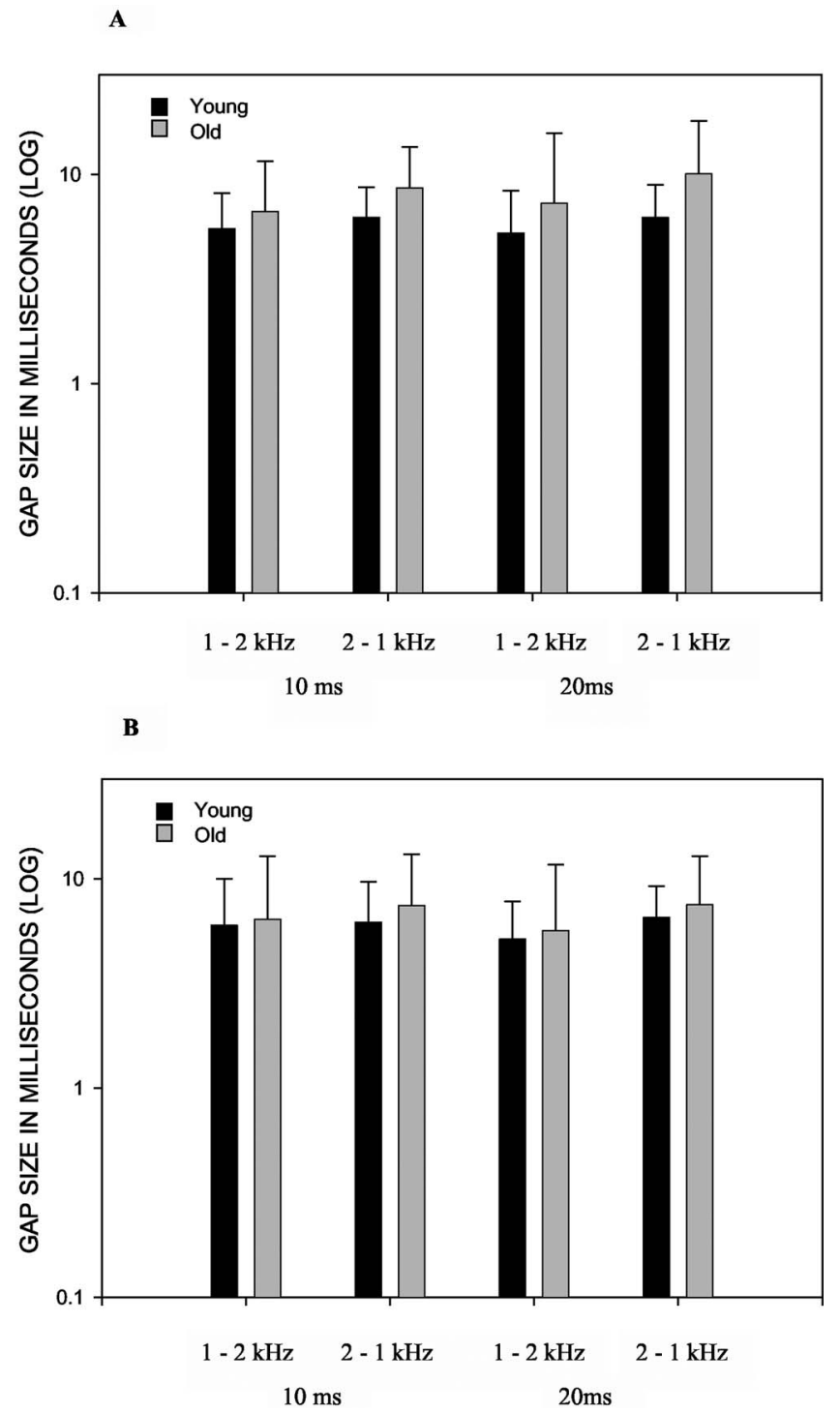

FIG. 3. (A) Mean gap detection thresholds for young (black) and old (gray) listeners for 10 and 20-ms markers of dissimilar frequency (between channel). Stimulus frequency either ascended $(1 \mathrm{kHz}$ in the first marker and $2 \mathrm{kHz}$ in the second marker) or descended. Panel (A) presents the thresholds for the condition in which the no-gap stimulus matched the gap stimulus in total duration. Panel (B) presents the thresholds for the condition in which the duration of the markers in the no-gap stimulus was the same as the duration of markers in the gap stimulus. Note that in condition A the duration of the no-gap stimulus co-varied with that of the gap stimulus while in condition B, the no-gap stimulus was constant throughout the session. Error bars depict 1 standard deviation above the mean.

pared to the 20-ms marker duration, even though the age difference reaches significance in both instances [10-ms duration: $t(46)=-3.62, p<0.001 ; 20-\mathrm{ms}$ duration: $t(46)$ $=-3.02, p<0.01]$. None of the other interactions were significant.

\section{B. Between-channel gap detection}

Figure 3 depicts gap detection thresholds for young and old listeners in between-channel conditions when the no-gap reference tone was (A) adjusted in each trial to match the overall duration of the gap stimulus or (B) fixed at the combined marker duration of the two markers in the gap stimulus. The data show age differences in both plots but these 
differences appear to be more pronounced in the matched condition than in the fixed condition. Moreover, there is a consistent advantage for gap thresholds when the marker frequencies before and after the gap are ascending from 1 to $2 \mathrm{kHz}$ compared to when they are descending. Lastly, thresholds are in general smaller in fixed marker conditions than in adjusted marker conditions.

A mixed factor ANOVA with marker duration $(10 \mathrm{~ms}$, $20 \mathrm{~ms}$ ), frequency order (ascending from $1 \mathrm{kHz}$ to $2 \mathrm{kHz}$ or descending), and duration cue (matched or fixed) as withinsubject variables and age as a between-subject variable revealed a significant main effect of frequency order $[F(1,46)$ $=27.92, p<0.0001]$ but no main effect of age, marker duration, or duration cue. The significant effect of frequency order confirms that thresholds are smaller for ascending than for descending frequency order. Moreover, there were three significant interaction effects, two of them involving the duration cue: between duration cue and age group $[F(1,46)$ $=4.97, p<0.05]$ and duration cue and marker duration $[F(1,46)=5.02 ; p<0.05]$, and between marker duration and frequency order $[F(1,46)=4.87, p<0.05]$. No other interactions were significant.

The interaction between duration cue and age reflects the fact that age differences in threshold were more pronounced when stimuli were adjusted for overall duration than when the overall duration of the reference tone was fixed. In fact, a significant age effect was only present when reference stimuli were matched in overall duration to the gap stimuli $[t(46)=-2.70, p=0.01]$, but not when the overall duration of the reference stimuli was fixed $[t(46)=-0.92, p$ $>0.05]$.

The interaction between duration cue and marker duration was caused by the fact that when the reference stimulus changed with gap size, gap thresholds were larger for 20-ms than for 10-ms markers. On the other hand, when the reference stimuli were fixed in overall duration, thresholds were smaller for 20-ms than for 10-ms markers. However, neither of these threshold changes were significant $[t(47)$ $=-1.17, p=0.25$ when the reference stimulus changed with gap size; $t(47)=1.31, p=0.20$, when the reference stimulus was fixed].

Lastly, the interaction between marker duration and frequency order was caused by the fact that, for an ascending frequency order, thresholds decreased in size when going from 10 - to 20-ms duration markers. In contrast, for the descending frequency order thresholds were larger when the markers were $20 \mathrm{~ms}$ in duration compared to $10 \mathrm{~ms}$. Yet, neither of these threshold changes at each marker duration reached significance [ascending frequency order: $t(47)$ $=1.26, p=0.21$; descending frequency order: $t(47)=-1.53, p$ $=0.13]$.

\section{Correlation between pure-tone thresholds and within-channel gap detection thresholds}

Lastly, in order to rule out that the age differences in the within-channel conditions were caused by age-related puretone threshold elevations, we computed Pearson productmoment correlations between listeners' gap detection thresh- olds for a particular frequency condition and their respective hearing level at that frequency. For instance, the correlation between individual gap detection thresholds for the $1-\mathrm{kHz}$ within-channel condition with 10-ms markers and hearing levels at $1 \mathrm{kHz}$ were -0.01 for young adults and -0.28 for old adults, respectively. Correlations were equally small for the other three within-channel conditions, ranging from 0.07 to 0.16 in young listeners and from -0.27 to -0.36 in old listeners. None of the correlations reached significance. Hence, there is no evidence that within-channel gap detection thresholds vary with hearing level.

To determine whether between-channel performance was related to hearing levels at each of the two frequencies, we correlated gap thresholds for each of eight conditions (see Table II) with audiometric thresholds at each of the two test frequencies. This yielded 16 correlation coefficients for younger adults and 16 correlation coefficients for older adults. Of the 32 correlations tested only one reached the alpha $=0.05$ level of significance. Under the null hypothesis that hearing level and gap detection thresholds were independent of each other we would expect $5 \%$ of the 32 correlation coefficients (or 1.6 correlation coefficients) to reach significance. Moreover, in the one case that reached significance (which occurred in older adults), the sign of the correlation indicated that gap detection thresholds improved as hearing levels worsened. Because of this, and because only one correlation coefficient turned out to be significant, there is no evidence to suggest that between-channel gap detection performance is correlated with hearing levels at either of the two frequencies.

\section{DISCUSSION}

\section{A. Within-channel gap detection}

Figure 2 and its associated ANOVA indicated that (1) older adults had larger gap detection thresholds than younger adults; (2) gap detection thresholds decreased for both younger and older adults as marker duration increased from 10 to $20 \mathrm{~ms}$; (3) the size of the age difference decreased as marker duration increased from 10 to $20 \mathrm{~ms}$; and (4) gap detection thresholds were lower for the $1-\mathrm{kHz}$ markers than they were for the $2-\mathrm{kHz}$ markers for both younger and older adults. The first three results are consistent with the findings from other within-channel gap detection studies that have used 1- or 2-kHz markers (Moore et al., 1992; Schneider et al., 1994, 1998; Schneider and Hamstra, 1999; Strouse et al., 1998). However, an effect of marker frequency on performance has not been consistently shown. Whereas some studies (Shailer and Moore, 1987; Moore and Glasberg, 1988; Formby and Forrest, 1991) found no appreciable difference in gap threshold for 1- and $2-\mathrm{kHz}$ marker frequencies, others (Moore et al., 1992) seemed to show that for both young and older normal listeners gap detection thresholds increased by about $1 \mathrm{~ms}$ when marker frequencies increased from 1 to $2 \mathrm{kHz}$. The results from the present study are in agreement with the latter results and show that an increase in marker frequency from 1 to $2 \mathrm{kHz}$ led to larger thresholds. 
Hence, there does appear to be a significant age difference in within-channel gap detection for short duration tonal markers that decreases with increasing marker duration and is uncorrelated with hearing loss.

\section{B. Between-channel gap detection}

Frequency order: A number of interesting results arose from the between-channel conditions. First, frequency order was the only significant main effect, whereas all other variables were only involved in interaction effects. The main effect of frequency order indicates that going from a $1-\mathrm{kHz}$ marker to a $2-\mathrm{kHz}$ marker resulted in smaller gap detection thresholds than when the $2-\mathrm{kHz}$ marker preceded the $1-\mathrm{kHz}$ marker. This result is in agreement with several studies that showed a similar asymmetry effect when the lagging marker has a higher frequency than the leading marker (Formby et al., 1996; Lister et al., 2000, 2002). However, other studies have not found an asymmetry in thresholds with respect to whether tonal frequency increased or decreased from the first to the second marker (Fitzgibbons et al., 1974; Formy et al., 1998b).

One possible explanation for this frequency order effect is harmonic distortion. If the $1-\mathrm{kHz}$ leading marker has distortion products at $2 \mathrm{kHz}$, then the participant could be basing her or his judgment on events occurring in the $2-\mathrm{kHz}$ channel, thereby turning a between-channel task into a within-channel task. To check whether the earphone tranducer was producing distortion products at $1 \mathrm{kHz}$, we recorded the sound pressure level produced by the earphones in the ear canal of a dummy head (Kemar), using the Bruel \& Kjaer Pulse platform. Those measurements showed that any harmonic distortion produced by the earphone was more than $70 \mathrm{~dB}$ down from the signal intensity. Hence, headphone distortion products cannot account for the observed effect.

However, it is possible that the presence of aural harmonics in the inner ear could have turned the betweenchannel task into a within-channel one. Aural harmonics or subjective tones refer to the phenomenon that, in the course of the sound transmission from the air to the basilar membrane, the ear sometimes introduces new frequencies into the original sound if the intensity of the pure tone is high enough (e.g., Wegel and Lane, 1924; Fletcher, 1930). For instance, Steinberg (1935) claims that any tone below $1 \mathrm{kHz}$ will produce noticeable harmonics when its intensity level exceeds $50 \mathrm{~dB}$. In fact, for high-intensity sounds, the intensity of the harmonics may exceed the magnitude of the fundamental (Steinberg, 1935). Lawrence and Yantis (1956) extended the demonstration of aural harmonics to frequencies of up to $5 \mathrm{kHz}$. Specifically, they determined that aural harmonics first occurred for a $1-\mathrm{kHz}$ tone at $50 \mathrm{~dB}$ SPL. At $70 \mathrm{~dB}$ SPL the second aural harmonic had an intensity of approximately $55 \mathrm{~dB}$ SPL. In the present case, the stimulus is a $1-\mathrm{kHz}$ tone whose level was approximately $70 \mathrm{~dB}$ SPL before it was multiplied by the marker envelopes. Hence, according to these measurements, we can assume that in addition to the original $1-\mathrm{kHz}$ frequency, sound energy was being generated by the ear for frequencies of $2 \mathrm{kHz}$ and possibly even higher harmonics. The second harmonic is of specific interest to us because if energy was present not only at 1 but also at $2 \mathrm{kHz}$, then the task may have acquired a within-channel component. Hence, rather than basing their evaluation of the temporal gap solely on the temporal characteristics of the 1- and $2-\mathrm{kHz}$ tone presentation, the listener may have also used within-channel cues provided by the second harmonic. When the first marker is a $1-\mathrm{kHz}$ tone it will generate an aural harmonic at $2 \mathrm{kHz}$. Hence, if the listener attends to the $2-\mathrm{kHz}$ channel, she or he should hear a weak $2-\mathrm{kHz}$ tone (the aural harmonic of the 1-kHz leading marker) when the leading marker is played, followed by a stronger $2-\mathrm{kHz}$ tone when the lagging marker (a $2-\mathrm{kHz}$ tone) is played. This would turn a between-channel task into a within-channel task. Divenyi and Danner (1977) found that the intensity of the leading marker could be as much as $25-45 \mathrm{~dB}$ lower than that of the second marker before it began to have a substantial effect on gap discrimination (identifying which of two gaps is larger). Hence, provided that the aural harmonic produced by the leading $1-\mathrm{kHz}$ tone in this experiment is sufficiently intense, participants could possibly base their judgments on events occurring in the $2-\mathrm{kHz}$ channel.

Although aural harmonics generated by a lagging $1-\mathrm{kHz}$ tone could also turn a between-channel task into a within-channel task, the effectiveness of monitoring the $2-\mathrm{kHz}$ channel is likely to be diminished because of forward masking effects. If the first marker is a $2-\mathrm{kHz}$ tone, the listener is presented with a strong $2-\mathrm{kHz}$ tone followed by the weaker, $2-\mathrm{kHz}$, aural harmonic generated by the lagging $1-\mathrm{kHz}$ tone. It has long been known that the presence of an intense masker elevates the threshold for a same-frequency probe stimulus following the masker (e.g., Duifhuis, 1973; McFadden and Yama, 1983; Nizami, 2003). Hence, in the ascending frequency case, the $2-\mathrm{kHz}$ tone is present as a weak-intensity aural harmonic in the leading marker and a $2-\mathrm{kHz}$ tone in the lagging marker. In the descending frequency case, the stronger 2-kHz tone is presented first and the weaker aural harmonic second. The data of Plomp (1964) and Penner (1977) show that it is much more difficult for observers to perceive a gap when the intensity of the first marker is greater than the intensity of the second marker than when the two markers have equal intensities. Thus the frequency-order asymmetry may result from a combination of the presence of aural harmonics at $2 \mathrm{kHz}$ coupled with forward-masking effects.

Marker duration: The data did not provide evidence for a general effect of marker duration for durations of 10 and $20 \mathrm{~ms}$. Although no studies have investigated the effects of marker duration on gap detection threshold for betweenchannel stimuli, at least one study has investigated the effect for within-channel stimuli. Schneider and Hamstra (1999) showed that age differences in within-channel gap detection thresholds declined as a function of marker duration, finally disappearing at the longest marker duration tested $(500 \mathrm{~ms})$.

Marker duration and frequency order: The interaction between marker duration and frequency order was caused by the fact that in the case of an ascending frequency order longer marker durations led to smaller gap thresholds whereas in the descending frequency order conditions the opposite was true. This result is consistent with the notion 
that, due to the presence of aural harmonics, the ascending frequency conditions contained a within-channel component and therefore changes in marker duration in these conditions had similar effects as in pure within-channel conditions.

Marker duration and duration cue: Previous withinchannel studies (e.g., Schneider and Hamstra, 1999) have indicated that gap thresholds decrease with increasing marker duration, a result that was replicated in our withinchannel conditions. In the between-channel case, gap thresholds also decreased with increasing marker duration when the overall duration of the reference stimulus was fixed. Thus, the effects of marker duration on gap detection thresholds are the same for both within- and between-channel conditions when the overall duration of the no-gap or reference stimulus in the between-channel conditions is fixed. However, when the duration of the reference stimulus was matched to that of the gap stimulus in the between-channel case, gap detection thresholds increased as the duration of the markers defining the gap changed from 10 to $20 \mathrm{~ms}$. This deviation from the expected pattern may reflect the fact that it is more difficult to determine the presence of a gap when the duration of the markers in the no-gap or reference stimulus are changing with the size of the gap.

Age: The only significant age effect in between-channel conditions occurred when the stimuli were matched for overall duration. This result is in stark contrast to the clear age differences that have been found in at least four betweenchannel gap detection studies (Lister et al., 2000, 2002; Pichora-Fuller et al., 2006, Roberts and Lister, 2004). We propose that the overall size of the gap thresholds as well as the size of the age effect is a function of the complexity of the stimuli. In this context stimulus complexity can be seen as a continuum where pure-tone markers represent the simplest form of gap markers that lead to the smallest overall thresholds and the smallest age differences. Markers consisting of harmonic complexes, noises, and speech sounds represent more complex sounds leading to generally higher thresholds and more pronounced age differences (Lister et al., 2000, 2002; Haubert and Pichora-Fuller, 1999).

Duration cue and age: For older adults, gap detection thresholds were lower in between-channel conditions when marker duration was fixed than they were when gap and no-gap stimuli were matched in overall duration. In contrast, in younger adults, the two types of marker duration cues had no effect on gap detection thresholds.

Because the testing sequence was always such that conditions with matched overall durations were tested prior to conditions with fixed marker durations, we tried to assess whether practice effects may have been responsible for the effect of duration cue on older adults. To assess the effects of practice on performance, we looked for evidence of improvements in performance as a function of the number of threedown, one-up tracking runs in each between-channel condition in an omnibus ANOVA with duration cue, marker duration, frequency order, and trial number as within-subject variables. There was no indication of a practice effect for young $[F(3,69)=1.71, p=0.1]$ or old adults $[F(3,69)<1$, $p=0.51]$, confirming that in neither age group did gap detection thresholds decrease with practice. Moreover, practice also did not interact with duration cue for young $[F(3,69)$ $<1, p=0.98]$ or old adults $[F(3,69)<1, p=0.98]$. Hence, it is unlikely that learning was responsible for the effect that the two types of duration cues had on older adults.

Because the gap detection thresholds of the older listeners were more variable in all between-channel conditions, it is possible that some older adults used duration cues while others did not, or that older listeners used duration cues in some runs but not in others. This interpretation would also be in agreement with Formby and Muir (1989) who found that different listeners used different cues at different times.

Another possibility is that the presence of two sets of discriminable cues (marker duration cues and gap cues) at the larger gap sizes was more confusing to older than to younger adults. Because our adaptive procedure started with gap sizes of $61 \mathrm{~ms}$, participants, when encountering these larger gap sizes, could base their decision on the difference in gap size ( 0 vs. $61 \mathrm{~ms}$ ), the difference in overall duration for the condition in which marker duration was fixed (20 vs. $81 \mathrm{~ms}$ for the 10-ms markers), or on marker duration when gap and no-gap stimuli were matched in overall duration (10 vs. $40 \mathrm{~ms}$ for the 10 -ms markers). Because age differences are more likely to appear when either the stimuli or the task is more complex, it is possible that some older adults performed less well than younger adults because of the additional complexity of the task. Moreover, one could argue that stimulus complexity was greater when the gap and no-gap stimuli were equated for overall duration than when the marker duration was fixed. In the former condition both the total duration of the no-gap stimulus and the duration of each of its markers covaried with gap duration. In the latter, the no-gap stimulus was unchanged from trial to trial. It is possible that older adults found it more difficult than younger adults to make the discrimination when the duration of the no-gap stimulus varied from trial to trial, but not when the no-gap reference stimulus remained unchanged over an adaptive run. It is important to note that such trial-by-trial changes in total duration are unlikely to affect performance in the within-channel task because the overall durations are so short that trial-by-trial variation in overall duration would not be noticeable near threshold levels.

Finally, there is always the possibility that temporal acuity was particularly well preserved in our sample of older adults and that this is the reason why we did not find any substantial age differences in between-channel tasks. However, the fact that the older adults in our sample performed worse than the younger adults in within-channel gap detection tasks, and that the pattern of age differences in withinchannel gap detection was consistent with previous studies, suggests that our group of older adults was not atypical with respect to temporal acuity. Hence, we conclude that age differences in between-channel tasks increase with stimulus complexity.

\section{SUMMARY}

Within-channel, but not between-channel, pure-tone gap detection thresholds were consistently higher for older than for younger adults. The failure to find substantial age differ- 
ences in between-channel gap detection in the present experiment stands in sharp contrast to a number of studies in which substantial age differences were reported (e.g., Lister et al., 2000; 2002; Pichora-Fuller et al., 2006; Roberts and Lister, 2004). However, those studies reporting substantial age effects have all used spectrally complex markers (noise bands and/or speech stimuli) to define a gap whereas our markers were pure tones. This suggests that age differences in gap detection are modulated by stimulus complexity, with greater complexity leading to greater age differences. The fact that age differences were larger in the between-channel condition of the present study when the duration of the no-gap stimulus varied over trials than when the no-gap stimulus was invariant over trials also suggests that age differences may be magnified by task complexity. Finally, the fact that betweenchannel performance was better when the first and second markers were 1 and $2 \mathrm{kHz}$, respectively, than when the same markers appeared in the reverse order is consistent with the notion that both younger and older adults can use the aural harmonics produced in the ear by the first marker to aid gap detection.

\section{APPENDIX: POWER SPECTRA FOR GAP AND NO-GAP STIMULI WITH EQUAL DURATION MARKERS}

A stimulus with a gap consists of two markers, a leading marker and a lagging marker with a gap between them. Consider the case where the leading and lagging markers have equal duration, with each marker consisting of a sinusoid multiplied by a temporal window. Let each temporal window consist of a sum of $n+1$ Gaussians spaced 1 standard deviation ( $\sigma$ seconds $)$ apart. Furthermore, assume that the first window is multiplied by a sinusoid whose frequency is $r_{1} / \sigma$ $\mathrm{Hz}$, and that the second window is multiplied by a sinusoid whose frequency is $r_{2} / \sigma \mathrm{Hz}$, where $r_{1}$ and $r_{2}$ are both integers greater than 0 . (In the present experiment $r_{1}=1, r_{2}=2$, $\sigma=0.001$ s.) Finally, a gap between the two markers is produced by centering the first Gaussian in the envelope of the second marker, $(m+1) \sigma$ seconds after the center of the last Gaussian in the first marker, where $m$ is an integer. Specifically, if $g_{1}(t)$ is the first marker, and $g_{2}(t)$ is the second marker, then

$$
\begin{aligned}
& g_{1}(t)=\cos \left(\frac{2 \pi r_{1} t}{\sigma}\right)\left[\sum_{k=0}^{n} e^{-(t-k \sigma)^{2} / 2 \sigma^{2}}\right], \\
& g_{2}(t)=\cos \left(\frac{2 \pi r_{2} t}{\sigma}\right)\left[\sum_{k=m+n+1}^{m+2 n+1} e^{-(t-k \sigma)^{2} / 2 \sigma^{2}}\right] .
\end{aligned}
$$

Note that if $m=0$, there is no gap between the first and second markers, that is, the sum of the envelopes of the two markers is flat during the switchover from frequency $r_{1} / \sigma$, to frequency $r_{2} / \sigma$. If $m$ is an integer greater than zero, there is a gap between the two markers equal to $(m+1) \sigma$.

Figures 1(a) and 1(b) show how a 13-ms gap is inserted between two 20-ms markers $(n=20, m=12)$. The standard deviation of the Gaussians shown in Fig. 1(a) is $1 \mathrm{~ms}$, and the means of the Gaussians are spaced $1 \mathrm{~ms}$ apart. Therefore the envelope for the first marker is obtained by summing the first 21 Gaussians in Fig. 1(a). If the center of the first Gaussian is at $T_{0} \mathrm{~ms}$, the center of the 21st Gaussian is at $T_{0}+n$ $\times \sigma=T_{0}+20 \mathrm{~ms}$. Hence the duration of the first marker is $20 \mathrm{~ms}$. The location of the first Gaussian in the second marker is set at $T_{0}+n \sigma+(m+1) \sigma=T_{0}+20+13 \mathrm{~ms}$. Therefore the duration of the gap is $13 \mathrm{~ms}$. The 21 Gaussians defining the envelope of the second marker begin at $T_{0}+n \sigma+(\mathrm{m}$ $+1) \sigma=T_{0}+20+13 \mathrm{~ms}$ and end at $T_{0}+2 n \sigma+(m+1) \sigma=T_{0}$ $+40+13 \mathrm{~ms}$. Hence the duration of the second marker is also $20 \mathrm{~ms}$. The envelopes of the two markers are obtained by summing the Gaussians associated with each marker. The results of this summation process are shown in Fig. 1(b) (the envelopes have been normalized by setting the maximum amplitude to 1.0).

Figures 1(c) and 1(d) show how the envelopes of the no-gap comparison stimulus are constructed for the condition in which the duration of the no-gap comparison stimulus is equal to the total duration of the gap stimulus. The total duration of the gap stimulus is $2 n \sigma+(m+1) \sigma \mathrm{ms}=53 \mathrm{~ms}$. The equal duration comparison stimulus is obtained by summing 54 Gaussians. The first 27 Gaussians define the envelope for the first marker. The second 27 Gaussians define the envelope of the second marker. The two summed envelopes are shown in Fig. 1(d).

Finally, Figs. 1(e) and 1(f) show how the envelope of the no-gap comparison is constructed for the condition in which the duration of each of the markers in the no-gap comparison stimulus is equal to the duration of each of the markers in the gap stimulus. The first 21 Gaussians are used to determine the envelope of the first marker, the second 21 Gaussians the envelope of the second marker. The two marker envelopes are shown in Fig. 1(f). For both types of comparison stimuli the sum of the two marker envelopes is flat during the transition period between the first and second markers.

To obtain the energy density function for $g_{1}(t)+g_{2}(t)$ we note that the Fourier transform of $g_{1}(t)$ is

$$
\begin{aligned}
& \int_{-\infty}^{\infty} \cos (2 \pi f t) \cos \left(\frac{2 \pi r_{1} t}{\sigma}\right)\left[\sum_{k=0}^{n} e^{-(t-k \sigma)^{2} / 2 \sigma^{2}}\right] d t \\
& -j \int_{-\infty}^{\infty} \sin (2 \pi f t) \cos \left(\frac{2 \pi r_{1} t}{\sigma}\right)\left[\sum_{k=0}^{n} e^{-(t-k \sigma)^{2} / 2 \sigma^{2}}\right] d t
\end{aligned}
$$

and the Fourier transform of $g_{2}(t)$ is

$$
\begin{aligned}
& \int_{-\infty}^{\infty} \cos (2 \pi f t) \cos \left(\frac{2 \pi r_{2} t}{\sigma}\right)\left[\sum_{k=m+n+1}^{m+2 n+1} e^{-(t-k \sigma)^{2} / 2 \sigma^{2}}\right] d t \\
& -j \int_{-\infty}^{\infty} \sin (2 \pi f t) \cos \left(\frac{2 \pi r_{2} t}{\sigma}\right)\left[\sum_{k=m+n+1}^{m+2 n+1} e^{-(t-k \sigma)^{2} / 2 \sigma^{2}}\right] d t .
\end{aligned}
$$

Hence the Fourier transform of $g_{1}(t)+g_{2}(t)$ is the sum of the quantities in (A3) and (A4). It can be shown ${ }^{3}$ that the spectral density function of $g_{1}(t)+g_{2}(t)$ [the square of this Fourier transform of $\left.g_{1}(t)+g_{2}(t)\right]$ is 


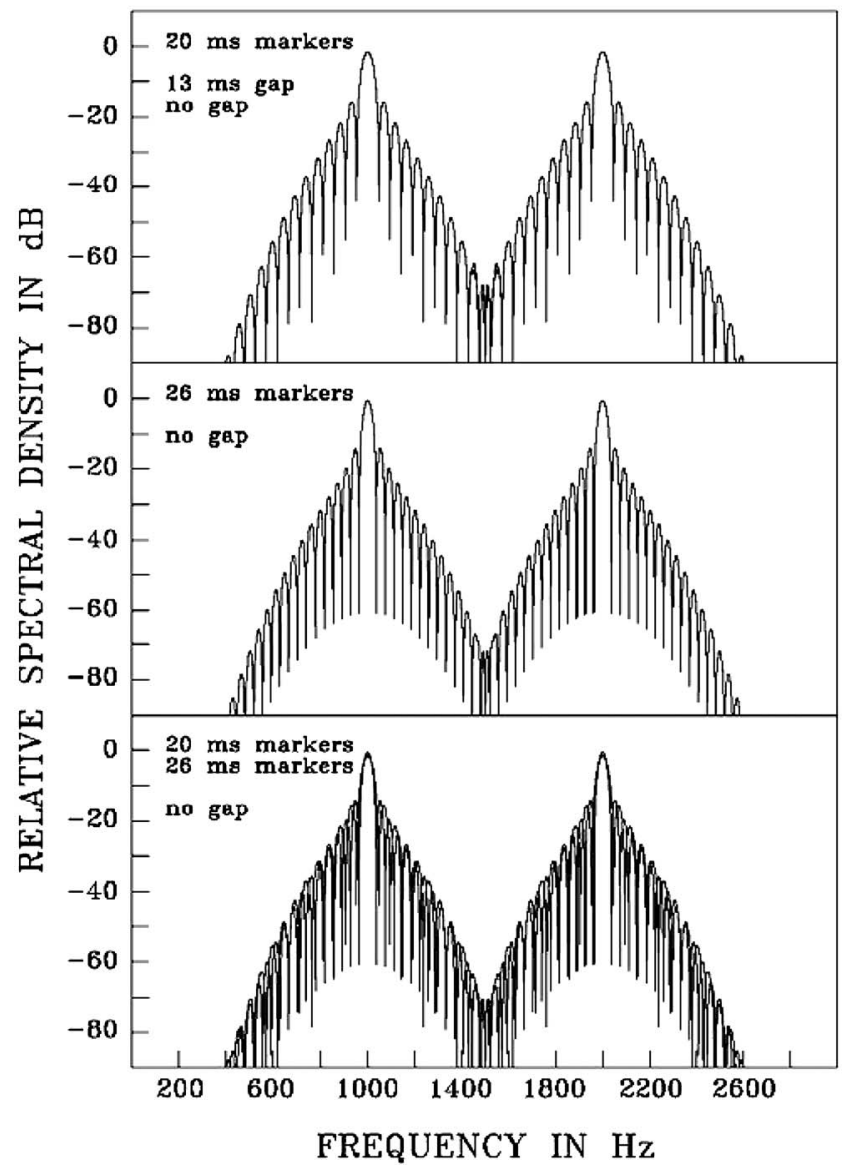

FIG. 4. Top panel: relative spectral densities of two 20-ms markers (one at $1 \mathrm{kHz}$, the other at $2 \mathrm{kHz}$ ) separated by a gap of $13 \mathrm{~ms}$ [see Figs. 1(a) and 1(b)], and the same two markers without a separation between them [the no-gap stimulus with equivalent marker durations, Figs. 1(e) and 1(f)]. Middle panel: the spectral density of the no gap stimulus whose total duration is equivalent to that of the stimulus with a 13-ms gap. Each marker duration is $26 \mathrm{~ms}$ long with a 1-ms separation between the end of the first marker and the start of the second [Figs. 1(c) and 1(d)]. Bottom panel: spectral densities of the two no-gap reference stimuli, one whose marker durations are equivalent to those in the gap stimulus $(20 \mathrm{~ms})$ and one whose marker durations are $26 \mathrm{~ms}$ each so that the total duration of the two markers is equivalent to that of the stimulus with the gap.

$$
\begin{aligned}
\frac{1}{2} \sigma^{2} & \pi \frac{\sin ^{2}((1+n) \pi f \sigma)}{\sin ^{2}(\pi f \sigma)} \\
\times & {\left[A^{2}+B^{2}+2 A B \cos (2(m+n+1) \pi f \sigma)\right], }
\end{aligned}
$$

where

$$
\begin{aligned}
& A=\left[e^{-2 \pi^{2} \sigma^{2}\left(f-r_{1} / \sigma\right)^{2}}+e^{-2 \pi^{2} \sigma^{2}\left(f+r_{1} / \sigma\right)^{2}}\right], \\
& B=\left[e^{-2 \pi^{2} \sigma^{2}\left(f-r_{2} / \sigma\right)^{2}}+e^{-2 \pi^{2} \sigma^{2}\left(f+r_{2} / \sigma\right)^{2}}\right] .
\end{aligned}
$$

Figure 4 plots the energy density functions (in decibels) for the gap and the two no-gap stimuli when $\sigma=0.001 \mathrm{~s}, r_{1}$ $=1$, and $r_{2}=2$, so the two frequencies in the cross-channel condition become 1 and $2 \mathrm{kHz}$, respectively, $n=20$, so that the two marker durations are each $20 \mathrm{~ms}$ long and $m=12$, thereby producing a gap of $13 \mathrm{~ms}$. The top panel of Fig. 4 plots the energy density functions for the stimulus with a 13-ms gap between two 20-ms markers [Fig. 1(b)], and for the no-gap comparison stimulus whose marker durations are $20 \mathrm{~ms}$ [Fig. 1(f)]. Note that except for the small region near
$1500 \mathrm{~Hz}$ these two energy density functions are essentially equal. The middle panel of Fig. 4 plots the energy density function for the no-gap comparison stimulus whose total duration is equal to that of the gapped stimulus. Note that the overall form of this spectral density function is quite close to that of the no-gap comparison stimulus whose marker durations are equal to those in the gap stimulus. This is illustrated in the bottom panel of Fig. 4 where the energy density functions of both comparison stimuli are shown. Therefore, Fig. 4 shows that it is unlikely that the discrimination between the gap stimulus and either of the two no-gap comparison stimuli is based on spectral cues.

${ }^{1}$ Consider a sum of Gaussians. When $m$ Gaussians are removed from that sum it creates a dip in the envelope. We can show that when $m$ Gaussians are removed from the middle of the envelope, the time difference between when the envelope decays to 0.7 of its peak value and when it returns to 0.7 of its peak amplitude is approximately equal to $(m+1) \sigma$. Since the 0.7 position on the amplitude envelope is approximately the 0.5 position on the squared envelope, the time difference between the half power points on the envelope is approximately equal to $(m+1) \sigma$ for $m$ greater than or equal to 1. The difference in area, $A$, between the full envelope and the envelope missing $m$ Gaussians is equal to $m \sqrt{2 \pi} \sigma$. Note that the time gap, $t_{g}$ (measured in seconds), created by the $m$ missing Gaussians is $t_{g}=(m+1) \sigma$, for $m$ greater than or equal to 1 . Therefore area is linearly related to time gap by $A=\left(t_{g}-\sigma\right) \sqrt{2 \pi}$ for $t_{g} \geqslant 2 \sigma, t_{g}$ measured in seconds. When there are no missing Gaussians, the gap duration is defined as 0 , and the area is 0 . When there is one missing Gaussian the gap duration is $2 \sigma$ and the area is $\sqrt{2 \pi} \sigma$. If we now assume that the area is linearly related to gap duration over this range, area $=(\sqrt{2 \pi} / 2) t_{g}$ measured in seconds, and $0<t_{g}<2 \sigma$. All analyses were performed on areas, and means were then converted back to equivalent gap durations using these linear relationships.

${ }^{2}$ Note that Fig. 2 shows gap thresholds in time units (ms) whereas area units were used for all statistical analyses. These area units were log transformed to keep the variances relatively constant over experimental conditions. The log-transformed mean area units were then translated back into time units. ${ }^{3}$ For a proof please contact Bruce A. Schneider at bschneid@utm.utoronto.ca

American National Standards Institute (1989). "Specification for audiometers (ANSI S3.6-1989)," New York: ANSI.

Bergeson, T. R., Schneider, B. A., and Hamstra, S. J. (2001). "Duration discrimination in younger and older adults," Can. Acoust. 29, 3-9.

Clark, J. G. (1981). "Uses and abuses of hearing loss classification," ASHA 23, 493-500.

Divenyi, P. L., and Danner, W. F. (1977). "Discrimination of time intervals marked by brief acoustic pulses of various intensities and spectra," Percept. Psychophys. 21, 125-142.

Duifhuis, H. (1973). "Consequences of peripheral frequency selectivity for nonsimultaneous masking," J. Acoust. Soc. Am. 54, 1471-1488.

Fitzgibbons, P. J., Pollatsek, A., and Thomas, I. B. (1974). "Detection of temporal gaps within and between perceptual tonal groups," Percept. Psychophys. 16, 522-528.

Fletcher, H. (1930). "A space-time pattern theory of hearing," J. Acoust. Soc. Am. 1, 311-343.

Formby, C., and Forrest, T. G. (1991). "Detection of silent temporal gaps in sinusoidal markers," J. Acoust. Soc. Am. 89, 830-837.

Formby, C., and Muir, K. (1989). "Effects of randomizing signal level and duration on temporal gap detection," Audiology 28, 250-257.

Formby, C., Sherlock, L. P., and Forrest, T. G. (1996). "An asymmetric roex filter model for describing detection of silent temporal gaps in sinusoidal markers," Aud. Neurosci. 3, 1-20.

Formby, C., Sherlock, L. P., and Li, S. (1998a). "Temporal gap detection measured with multiple sinusoidal markers: Effects of marker number, frequency, and temporal position,” J. Acoust. Soc. Am. 104, 984-998.

Formby, C., Gerber, M. J., Sherlock, L. P., and Magder, L. S. (1998b). "Evidence for an across-frequency, between-channel process in asymptotic monaural temporal gap detection,” J. Acoust. Soc. Am. 103, 3554-3560. Grose, J. H., Hall, J. W., III, Buss, E., and Hatch, D. (2001). "Gap detection 
for similar and dissimilar gap markers," J. Acoust. Soc. Am. 109, 15871595.

Haubert, N., and Pichora-Fuller, M. K. (1999). "The perception of spoken language by elderly listeners: Contribution of auditory temporal processes," Can. Acoust. 27, 96-97.

Lawrence, M., and Yantis, P. A. (1956). "Onset and growth of aural harmonics in the overloaded ear," J. Acoust. Soc. Am. 28, 852-858.

Levitt, H. (1971). "Transformed up-down methods in psychoacoustics," J. Acoust. Soc. Am. 49, 467-477.

Lister, J., Besing, J., and Koehnke, J. (2002). "Effects of age and frequency disparity on gap duration discrimination," J. Acoust. Soc. Am. 111, $2793-$ 2800.

Lister, J., Koehnke, J., and Besing, J. (2000). "Binaural gap duration discrimination in listeners with impaired hearing and normal hearing," Ear Hear. 21, 141-150.

Lutman, M. E. (1991). "Degradations in frequency and temporal resolution with age and their impact on speech identification," Acta Oto-Laryngol. 476, 120-126.

McFadden, D., and Yama, M. F. (1983). "Upward shifts in the masking pattern with increasing masker intensity," J. Acoust. Soc. Am. 74, 11851189.

Moore, B. C. J., and Glasberg, B. R. (1988). "Gap detection with sinusoids and noise in normal, impaired, and electrically stimulated ears," J. Acoust. Soc. Am. 83, 1093-1101.

Moore, B. C. J., Peters, R. W., and Glasberg, B. R. (1992). "Detection of temporal gaps in sinusoids by elderly subjects with and without hearing loss," J. Acoust. Soc. Am. 92, 1923-1932.

Moore, B. C. J., Glasberg, B. R., Donaldson, E., McPherson, T., and Plack, C. J. (1989). "Detection of temporal gaps in sinusoids by normally hearing and hearing-impaired subjects," J. Acoust. Soc. Am. 85, 1266-1275.

Nizami, L. (2003). "Afferent response parameters derived from postmasker probe-detection thresholds: 'the decay of sensation' revisited," Hear. Res. $175,14-35$.

Penner, M. J. (1977). "Detection of temporal gaps in noise as a measure of the decay of auditory sensation," J. Acoust. Soc. Am. 61, 552-557.

Phillips, D. P., Hall, S. E., Harrington, I. A., and Taylor, T. L. (1998). "“Central' auditory gap detection: A spatial case," J. Acoust. Soc. Am. 103, 2064-2068.
Phillips, D. P., Taylor, T. L., Hall, S. E., Carr, M. M., and Mossop, J. E. (1997). "Detection of silent intervals between noises activating different perceptual channels: Some properties of 'central' auditory gap detection," J. Acoust. Soc. Am. 101, 3694-3705.

Pichora-Fuller, M. K. (2003). "Processing speed and timing in aging adults: Psychoacoustics, speech perception, and comprehension," Int. J. Audiol. 42, S59-S67.

Pichora-Fuller, K., Schneider, B. A., Benson, N., Hamstra, S., and Storzer, E. (2006). "Effect of age on detection of gaps in speech and non-speech markers varying in duration and spectral symmetry," J. Acoust. Soc. Am. 119, 1143-1155.

Plomp, R. (1964). "Rate of decay of auditory sensation," J. Acoust. Soc. Am. 36, 277-282.

Roberts, R. A., and Lister, J. L. (2004). "Effects of age and hearing loss on gap detection and the precedence effect: Broadband stimuli," J. Speech Lang. Hear. Res. 47, 965-978.

Schneider, B. A., and Hamstra, S. J. (1999). "Gap detection thresholds as a function of tonal duration for younger and older listeners," J. Acoust. Soc. Am. 106, 371-380.

Schneider, B. A., Speranza, F., and Pichora-Fuller, M. K. (1998). "Agerelated changes in temporal resolution: Envelope and intensity effects," Can. J. Exp. Psychol. 52, 184-190.

Schneider, B. A., Pichora-Fuller, M. K., Kowalchuk, D., and Lamb, M. (1994). "Gap detection and the precedence effect in young and old adults," J. Acoust. Soc. Am. 95, 980-991.

Shailer, M. J., and Moore, B. C. J. (1987). "Gap detection and the auditory filter-Phase effects using sinusoidal stimuli," J. Acoust. Soc. Am. 81, $1110-1117$.

Snell, K. B. (1997). "Age-related changes in temporal gap detection," J. Acoust. Soc. Am. 101, 2214-2220.

Steinberg, J. C. (1935). "Discussion from the point of the physicist," Ann. Otol. Rhinol. Laryngol. 44, 819-822.

Strouse, A., Ashmead, D. H., Ohde, R. N., and Grantham, D. W. (1998).

"Temporal processing in the aging auditory system," J. Acoust. Soc. Am. 104, 2385-2399.

Wegel, R. L., and Lane, C. E. (1924). "The auditory masking of one pure tone by another and its probable relation to the dynamics of the inner ear," Phys. Rev. 23, 266-285. 